\title{
All-Optical Regeneration Based on Phase-Sensitive Nondegenerate Four-Wave Mixing in Optical Fibers
}

\author{
Alexandros Fragkos, Adonis Bogris, and Dimitris Syvridis
}

\begin{abstract}
An optical regeneration scheme based on nondegenerate phase-sensitive amplification in highly nonlinear fibers is numerically investigated. The proposed regenerator exhibits significant amplitude and phase noise suppression properties and eliminates the need for precise phase and frequency locking with the input return-to-zero differential phase-shift keying signal.
\end{abstract}

Index Terms-Phase modulation, phase-sensitive amplification, return-to-zero to nonreturn-to-zero (RZ-to-NRZ) optical conversion.

\section{INTRODUCTION}

A DVANCED modulation formats such as differential phase-shift keying (DPSK) currently emerge as an alternative to on-off keying (OOK) for long-haul transmission. The superiority of DPSK is based on two fundamental advantages: The first one originates from the balanced detection of DPSK signals which offers a 3-dB improvement in receiver sensitivity over direct detection of OOK. The second one is its enhanced tolerance to fiber nonlinearities and basically to interchannel cross-phase modulation (XPM), although nonlinear phase noise is the major limitation for its performance.

The potential for commercial deployment of DPSK-based modulation formats has triggered worldwide the research activity on the optical manipulation of signals modulated in phase. As far as the optical regeneration is concerned, there have been proposed various ideas based on nonlinear amplifying loop mirrors (NALMs) [1], phase insensitive limiters [2], and phase sensitive amplifiers (PSAs) [3]. However, the phase-preserving limiters can only prevent the nonlinear phase noise build-up and cannot remove the accumulated phase noise. Among the proposed schemes, phase-sensitive amplification emerges as one of the most powerful processing tools acting directly at the phase of the optical field. Although PSA has the ability to perfectly squeeze the noise impairing the phase, its practical implementation is very challenging and has to take care of the precise locking of the frequencies and phases of the interacting waves

Manuscript received May 14, 2010; revised August 31, 2010; accepted October 11, 2010. Date of publication October 21, 2010; date of current version November 24, 2010. The research leading to these results was supported by the European Communities Seventh Framework Programme FP/2007-2013 under Grant 224547 (STREP PHASORS), and by the Iraklitos grant of Hellenic Ministry of Education and 216863 (BONE).

A. Fragkos and D. Syvridis are with the Optical Communications Laboratory, Department of Informatics and Telecommunications, University of Athens, Athens GR-15784, Greece (e-mail: alx_f@di.uoa.gr; dsyvridi@di.uoa.gr).

A. Bogris is with Optical Communications Laboratory, Department of Informatics and Telecommunications, University of Athens, Athens GR-15784, Greece, and also with the Department of Informatics, Technological Educational Institute of Athens, 12210 Egaleo, Athens, Greece (e-mail: abogris@ teiath.gr).

Color versions of one or more of the figures in this letter are available online at http://ieeexplore.ieee.org.

Digital Object Identifier 10.1109/LPT.2010.2089442

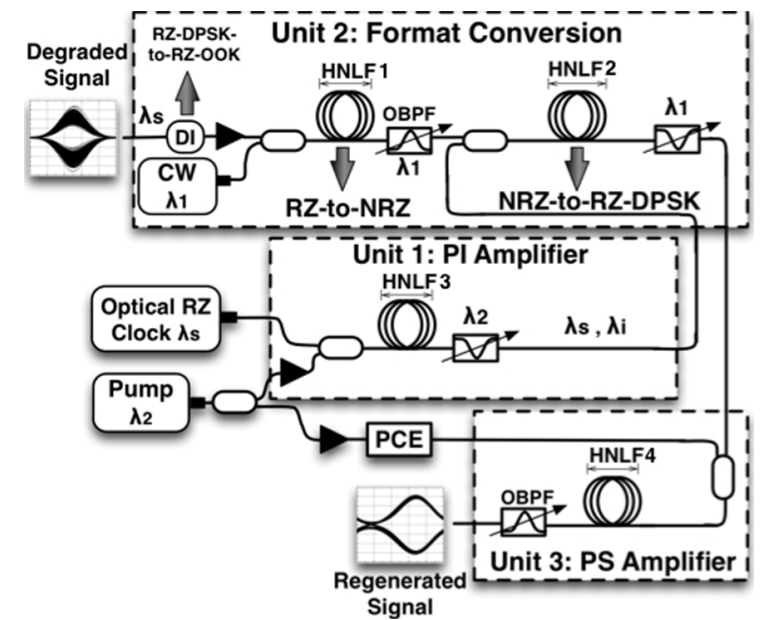

Fig. 1. Block diagram of the all-optical DPSK regenerator. DI: Delay interferometer; OBPF: optical bandpass filter; PCE: phase control element.

in advance. The regeneration experiments of [3] provided the proof of principle of amplitude and phase regeneration in interferometric and four-wave mixing (FWM)-based PSAs, but are far from being practical solutions, because the pump and the signal originated from the same laser and the phase modulation was applied after the phase locking process. Only very recently, a phase-sensitive amplifier utilizing injection locking and FWM was successfully demonstrated as the first back box regenerator based on a degenerate PSA [4]. The regenerator presented in [4] is mostly suitable for nonreturn-to-zero (NRZ)-DPSK signals as the locking process is based on injection locking a local laser with a constant envelope signal. In this letter, we propose a return-to-zero (RZ)-DPSK regenerator scheme (Fig. 1) based on a nondegenerate PSA. The proposed scheme embodies two novel approaches in comparison with the up to date reported PSA techniques. It relies on the nondegenerate FWM-based phasesensitive amplification which has the potential to offer multiwavelength amplification and it eliminates the need for precise frequency and phase locking with the input signal.

\section{PRINCIPLE OF OPERATION}

The scheme consists of three different units (Fig. 1). The first unit prepares an auxiliary signal and an idler through a typical single-pump FWM process in a highly nonlinear fiber (HNLF). The first unit is a typical fiber-optic parametric phase insensitive amplifier (PIA). The auxiliary signal of the FWM process is a typical clock of Gaussian pulses (the typical amplitude pattern of RZ-DPSK signals) with repetition rate equal to the bit-rate of the degraded input. By using the first FWM module, we obtain three frequency and phase-locked waves whereas signal and idler are two identical RZ clocks. The data of the input RZ-DPSK signal must be loaded on the phase of both auxiliary 
signal and idler with the same polarity. The latter is a prerequisite in order for both signal and idler to experience phase-sensitive amplification accompanied with phase noise squeezing as it will be explained later on.

The second unit undertakes the input signal data transfer onto the phase of the auxiliary signal and its idler. Initially, the second unit of the regenerator carries out the conversion of the signal phase information to amplitude. The incoming RZ-DPSK signal $\left(\lambda_{s}\right)$ passes through a delay interferometer (DI) which demodulates it to return an RZ-OOK bit stream. The next step includes the conversion of the RZ-OOK to an NRZ bit sequence as the typical RZ-DPSK modulation format encloses NRZ phase modulation pattern. This step is accomplished with the use of a second XPM stage where the RZ-OOK bit stream is combined with a continuous wave $(\mathrm{CW})\left(\lambda_{1}\right)$ [5]. The RZ phase modulation transferred to $\lambda_{1}$ leads to red and blue shifts of the frequency of the $\mathrm{CW}$. This wave is then filtered with a narrow bandwidth optical bandpass filter centered at its wavelength which converts the CW to NRZ pulses. The NRZ bit stream is an inversed replica of the RZ-OOK stream [5]. At the final step, the NRZ-OOK copy of the input signal, the auxiliary signal and the idler are coupled into a second XPM module (HNLF) in order to complete the transfer of the NRZ pulses to the phase of the RZ clocks. The power of the NRZ-OOK bit stream must be properly adjusted-taking into account the nonlinear medium parameters - in order to obtain a phase difference equal to $\pi$ between the two phase states of the auxiliary signal and its corresponding idler. After this process, auxiliary signal and idler are transformed to two identical, phase and frequency-locked RZ-DPSK waveforms.

The third unit embodies the phase sensitive amplification of the two RZ-DPSK waves. The carriers of the auxiliary signal, the idler, and the pump are advance locked (unit 1 task) with their phase relation dictated by $\phi_{s}+\phi_{i}-2 \phi_{p}=$ constant, where $\phi_{s}, \phi_{i}$, and $\phi_{p}$ are the phases of the auxiliary signal, the idler, and the pump. The data are applied to signal and idler with the same polarity after the phase-locking process (similar to [3]) utilizing unit 2 and the phase relation changes to $\phi_{s}+\phi_{d}+\phi_{i}+\phi_{d}-2 \phi_{p}=$ constant $+2 \phi_{d}$, where $\phi_{d}$ is the data modulation term. The previous relation shows that gain maxima occur at $\phi_{d}$ values that are multiples of $\pi$ equivalently to the degenerate case [3]. In order to acquire the maximum gain at the two states of " 0 " and " $\pi$ " of the phase modulation, one phase control element should track the relative phase. A piezo-electric transducer could play the role of a dynamic phase controller in a typical experiment [3]. At gain maxima, both RZ-DPSK waveforms will exhibit a significant reduction of the phase noise and wide-open final RZ-DPSK eye-diagrams. The phase noise squeezing capability of the PSA is dependent on the gain properties. The experiments of [3] and [4] have shown that PSA gain ratios greater than $15 \mathrm{~dB}$ are sufficient in order to provide the quantization of the output phase noise. The device could be exploited in a three fold manner working either as an in line amplifier-regenerator or a preamplifier at the detection level (the output is the auxiliary signal) or even as a wavelength converter-regenerator (the output is the regenerated idler).

\section{RESULTS AND DISCUSSION}

The nonlinear medium in this work is a typical HNLF. The HNLF transmission is simulated by numerically solving the nonlinear Schrödinger equation (NLSE) with the use of the split-step Fourier (SSF) method. The model accounts for up to the fourth-order dispersion. Moreover, in order to encounter the phase noise of all the waves participating in the scheme, and to highlight the independence of the proposed scheme on the input signal phase and frequency, rate equation model for the simulation of semiconductor lasers has been utilized [6]. Typical arbitrary semiconductor lasers with linewidth equal to $500 \mathrm{kHz}$ and relative intensity noise of $-140 \mathrm{~dB} / \mathrm{Hz}$ are considered. The auxiliary signal is a train of Gaussian pulses with pulsewidth equal to $6.25 \mathrm{ps}$ and repetition rate equal to $40 \mathrm{GHz}$. The wavelengths of pump $\left(\lambda_{2}\right)$, CW $\left(\lambda_{1}\right)$, and signal $\left(\lambda_{s}\right)$ are chosen to be equal to 1556,1525 , and $1536 \mathrm{~nm}$, respectively. For the RZ-to-NRZ optical conversion, a 1-km-long HNLF (HNLF1) with nonlinear parameter $\gamma=12 \mathrm{~W}^{-1} \cdot \mathrm{km}^{-1}$, fiber losses $a=0.7 \mathrm{~dB} / \mathrm{km}$, zero dispersion wavelength $\lambda_{0}=1540 \mathrm{~nm}$ and dispersion slope $d D / d \lambda=0.03 \mathrm{ps} / \mathrm{nm}^{2} / \mathrm{km}$ was considered. Unit1 and unit3 of the scheme rely on a 500-m-long HNLF (HNLF3, HNLF4) with $\lambda_{0}=1555 \mathrm{~nm}$. The pump power in each stage is equal to $27 \mathrm{dBm}$ providing small signal gain equal to 18 and $24 \mathrm{~dB}$ for unit1 and unit3, respectively. The fiber considered for the NRZ-OOK to RZ-DPSK conversion is $350 \mathrm{~m}$ long (HNLF2) with $\lambda_{0}=1555 \mathrm{~nm}$. The EDFAs are modeled as constant gain amplifiers that induce additive Gaussian noise with noise figure equal to $5 \mathrm{~dB}$. Each EDFA is accompanied by an optical bandpass Gaussian filter with $160-\mathrm{GHz} 3-\mathrm{dB}$ bandwidth. The output of the DI is amplified to $26.9 \mathrm{dBm}$ and coupled with a $25.72-\mathrm{dBm} \mathrm{CW}$ for the RZ-to-NRZ conversion. At the output of the format conversion unit the CW is filtered by a bandpass optical filter with $40-\mathrm{GHz}$ 3-dB bandwidth.

Initially, the distortion of the amplitude and phase characteristics of the RZ-DPSK input was carried out by simulating its fiber transmission through an 800-km-long transmission link. The link consists of typical single-mode fibers $\left(L_{\mathrm{SMF}}=40 \mathrm{~km}, a_{\mathrm{SMF}}=0.22 \mathrm{~dB} / \mathrm{km}, \gamma_{\mathrm{SMF}}=\right.$ $\left.1.3 \mathrm{~W}^{-1} \cdot \mathrm{km}^{-1}, \beta_{2}=-20 \mathrm{ps}^{2} / \mathrm{km}\right)$ and dispersion-compensating fibers $\left(L_{\mathrm{DCF}}=40 \mathrm{~km}, a_{\mathrm{DCF}}=0.27 \mathrm{~dB} / \mathrm{km}\right.$, $\left.\gamma_{\mathrm{DCF}}=4.5 \mathrm{~W}^{-1} \cdot \mathrm{km}^{-1}, \beta_{2}=20 \mathrm{ps}^{2} / \mathrm{km}\right)$ and optipcal amplifiers with noise figure equal to $5 \mathrm{~dB}$ which are placed periodically every $80 \mathrm{~km}$. The mean power of the RZ-DPSK signal at the input of the link was $0 \mathrm{dBm}$. Fig. 2(a) shows the original output of the DI (RZ-DPSK to RZ-OOK conversion) which shows the noise impaired signal and Fig. 2(b) shows the result of the RZ-to-NRZ conversion. The subsequent eye-diagram in Fig. 2(c) corresponds to the phase content of the auxiliary signal after the XPM interaction between the signal, the idler, and the NRZ replica of the input RZ-DPSK. The power of the NRZ (mean power equal to $22.7 \mathrm{dBm}$ ) and the fiber length and nonlinearity $\left(350 \mathrm{~m}, 12 \mathrm{~W}^{-1} \cdot \mathrm{km}^{-1}\right)$ are properly selected so as to convert the NRZ amplitude modulation into an NRZ phase modulation with modulation depth equal to $\pi$, through the XPM process. The eye-diagram of Fig. 2(d) is the phase content of the auxiliary signal after the PSA process. Eye-diagrams and histograms for level " 1 " (level " 0 " is similar) in Fig. 3 for a heavily impaired signal show the remarkable noise squeezing performance of the saturated PSA.

Depending on the auxiliary signal power, the PSA can operate either in the linear or in the saturation regime. The regenerator performance is investigated at both regimes for different 


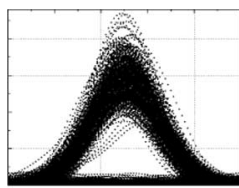

Time (5ps/div)

(a)

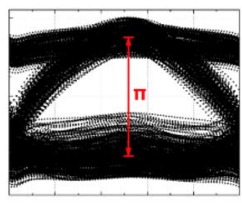

Time (5ps/div)

(c)

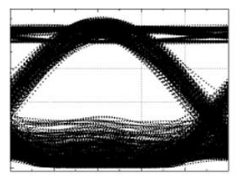

Time (5ps/div)

(b)

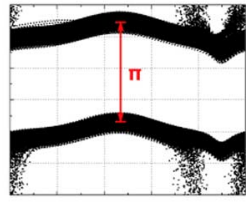

Time (5ps/div)

(d)
Fig. 2. (a) Eye-diagrams of the RZ-OOK signal after DI, (b) NRZ after format conversion at unit 2, (c) auxiliary signal phase before regeneration, and (d) after regeneration.

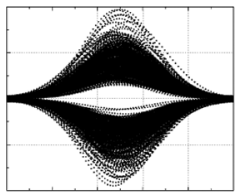

Time (5ps/div)

(a)

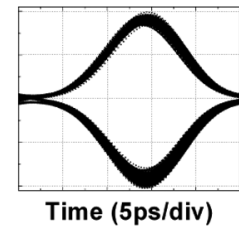

(b)

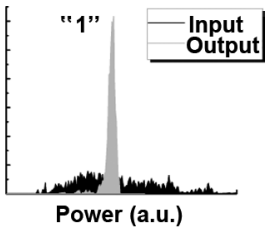

(c)
Fig. 3. (a) Eye-diagrams before, (b) after regeneration, and (c) corresponding histograms.

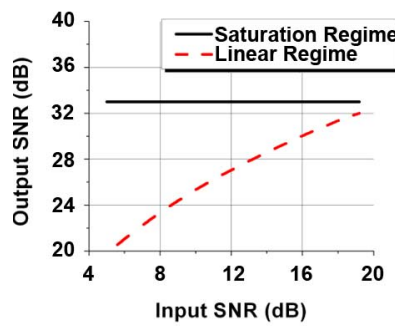

(a)

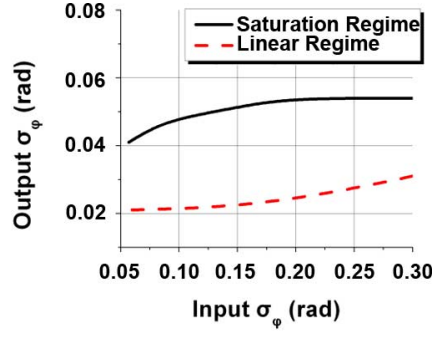

(b)
Fig. 4. (a) Output electrical SNR as a function of the input SNR and (b) output versus input phase standard deviation. Solid black lines correspond to the saturation regime while dashed red lines correspond to the linear regime.

noise levels of the initial signal. In this analysis, the signal distortion is simulated by adding white Gaussian noise of variable levels at the signal field (linear phase noise). The noise performance is quantified through the estimation of the electrical signal-to-noise ratio (SNR) of the RZ clocks and the standard deviation of the phase noise at the input and the output of the black box regenerator. The general impression derived from Fig. 4 is that the saturation regime outperforms linear regime in the amplitude regeneration, while the linear regime is slightly advantageous in terms of phase noise suppression. This was experimentally verified in [3]. However, it must be noted that at the input of the PSA (unit 3), the auxiliary signal and its corresponding idler are phase modulated signals with minor intensity noise because the RZ clocks are prepared locally at unit 1 . Due to the format conversions, the noise is impairing only their phase content. Therefore, the output SNR of the RZ clocks is expected to be high in both linear and saturation regimes and limited by the SNR of the clocks generated at unit 1 ( $\sim 32 \mathrm{~dB}$ in our setup).
The fact that the output SNR is worse in the linear regime is attributed to phase to amplitude conversions taking place inside the unsaturated PSA. Hence, part of the phase noise is transferred to the amplitude of the output RZ-DPSK. This effect is less evident in the saturation regime, as the gain is almost flattened close to its maximum value, hence different signal phases experience almost the same phase sensitive gain for a wide range of phase values [3]. The fact that the inputs of the PSA are subjected only to phase noise is another advantage of this approach. The research activity worldwide has shown that the PSA is capable of handling more efficiently the phase noise instead of the amplitude noise of a data stream [3], [4]. Hence, in order to fully exploit the regenerative properties of a PSA, it is desirable to transfer the amplitude noise of the input signal to its phase. The proposed scheme achieves so through the successive format conversions.

\section{CONCLUSION}

An optical regenerator based on a nondegenerate phase-sensitive amplifier has been proposed as an alternative solution with no need for cumbersome phase locking circuitry. The proposed scheme exploits the phase and frequency locking properties of FWM, the modulation format conversion potential of XPM, and the phase regeneration properties of PSAs. All the processes described in this letter were based on highly nonlinear fibers. A more practical scenario should incorporate short length semiconductor devices so as to prevent the loss of phase locking between signal, idler, and the pump. The RZ-to-NRZ conversion could be easily implemented in a semiconductor optical amplifier (SOA) [7] and the subsequent section of NRZ-OOK to RZ-DPSK conversion can be implemented in SOA Mach-Zehnder interferometers [8]. Future work will evaluate the same scheme with SOA-based devices for the implementation of the second unit.

\section{REFERENCES}

[1] C. Stephan, K. Sponsel, G. Onishchukov, B. Schmauss, and G. Leuchs, "Nonlinear phase noise reduction in a DPSK transmission system using cascaded nonlinear amplifying loop mirror," IEEE Photon. Technol. Lett., vol. 21, no. 24, pp. 1864-1866, Dec. 15, 2009.

[2] M. Matsumoto and K. Sanuki, "Performance improvement of DPSK signal transmission by a phase-preserving amplitude limiter," Opt. Express, vol. 15, no. 13, pp. 8094-8103, Jun. 2007.

[3] K. Croussore and G. Li, "Phase and amplitude regeneration of differential phase-shift keyed signals using phase-sensitive amplification," IEEE J. Sel. Topics Quantum Electron., vol. 14, no. 3, pp. 648-654, May/Jun. 2008.

[4] F. Parmigiani et al., "All-Optical Phase Regeneration of 40 Gbit/s DPSK Signals in a Blackbox Phase Sensitive Amplifier," in Proc. Optical Fiber Communication Conf. (OFC/NFOEC 2010), San Diego, CA, 2010, Paper PDPC3.

[5] S. H. Lee, K. K. Chow, and C. Shu, "Spectral filtering from a crossphase modulated signal for RZ to NRZ format and wavelength conversion," Opt. Express, vol. 413, no. 5, pp. 1710-1715, Mar. 2005.

[6] J. Wang and K. Petermann, "Noise analysis of semiconductor lasers within the coherence collapse regime," IEEE J. Quantum Electron., vol. 21, no. 1, pp. 3-9, Jan. 1991.

[7] T. Solveira, A. Ferreira, D. Fonseca, A. Teixeira, and P. Monteiro, "40 Gb/s all-optical RZ to NRZ format converter based on SOA and detuned filtering," in Proc. 11th Int. Conf. Transparent Optical Networks, 2009 (ICTON '09), Jun. 28-Jul. 2, 2009, pp. 1-4.

[8] K. Mishina et al., "NRZ-OOK-to-RZ-BPSK modulation-format conversion using SOA-MZI wavelength converter," J. Lightw. Tech., vol. 24, no. 10, pp. 3751-3758, Oct. 2006. 